\title{
Identification of Pathogenic Genes of Nonsyndromic Hearing Loss in Uyghur Families Using Massively Parallel DNA Sequencing Technique
}

\author{
Yu Chen, ${ }^{1}$ Yu Lu, ${ }^{2}$ Pilidong Kuyaxi, ${ }^{1}$ Jing Cheng, ${ }^{2}$ Juan Zhao, ${ }^{1}$ Qi Zhao, ${ }^{1}$ Patiguli Musha, ${ }^{3}$ \\ Hua Zhang $\left(\mathbb{D},{ }^{1}\right.$ and Huijun Yuan $\mathbb{1}^{2}$ \\ ${ }^{1}$ Department of Otorhinolaryngology, The First Affiliated Hospital of Xinjiang Medical University, Urumqi 830054, China \\ ${ }^{2}$ Medical Genetics Center, The First Affiliated Hospital, Army Medical University, Chongqing 400038, China \\ ${ }^{3}$ Department of Otorhinolaryngology, The First People's Hospital, Kashi Municipality, Xinjiang 844000, China \\ Correspondence should be addressed to Hua Zhang; hzhang106@163.com and Huijun Yuan; yuanhj301@163.com
}

Received 15 June 2017; Revised 28 August 2017; Accepted 28 September 2017; Published 5 March 2018

Academic Editor: Silvia Angeletti

Copyright @ 2018 Yu Chen et al. This is an open access article distributed under the Creative Commons Attribution License, which permits unrestricted use, distribution, and reproduction in any medium, provided the original work is properly cited.

\begin{abstract}
We aim to identify the mutations of deafness genes using massively parallel DNA sequencing in the 12 Uyghur families. SNPscan method was used to screen against the 124 sites in the common deafness genes in probands. Subjects with SNPscan negativity were subject to massively parallel DNA sequencing for the sequencing of 97 genes known to be responsible for hearing loss. Eight families (66.7\%) showed biallelic mutations in probands, including MYO15A mutation (6892C $>T$ in J02 family, 9514C $>$ T/7894G $>T$ in J07 family, and $9514 C>T$ in J16 family), MYO7A mutation (1258A $>T$ in J03 family), TMC1 mutation ( $773 G>A$ in $J 09$ family and $1247 T>G / 1312 G>A$ in J11 family), and PCDH15 mutation (4658delT in J08 and J13 families). Six novel types of mutation were identified including $6892 \mathrm{C}>T, 9514 \mathrm{C}>\mathrm{T} / 7894 \mathrm{G}>\mathrm{T}$, and $9514 \mathrm{C}>\mathrm{T}$ in $M Y O 15 \mathrm{~A}$ gene, $1258 \mathrm{~A}>\mathrm{T}$ in MYO7A, 773G $>A$ in TMC1, and 4658delT in PCDH15. The ratio of nonsense mutation and frameshift mutation was comparatively high. All these indicated that the mutation types reported in this study were rare. In conclusion, rare deafness genes were identified in the Uyghur families using massively parallel DNA sequencing, part of which were suggested to be related to the pathogenesis of the disease.
\end{abstract}

\section{Introduction}

Hereditary hearing loss is a highly heterogeneous disease with autosomal recessive nonsyndromic hearing loss (ARNSHL) being the most frequent condition occurring in $70 \%$ of the cases and syndromic in the other $30 \%$ [1]. Up to now, mutation in GJB2, SLC26A4, and $12 S$ rRNA has been considered as the major cause for the Han Chinese with ARNSHL $[2,3]$.

The genetic etiology of hearing loss may vary in different countries or races. As previously described, the positive rates of common hearing loss genes in the Uyghur minority were significantly lower than those of the Han Chinese (13.06-14.44\% versus $32.45-34.05 \%$ ), indicating that those two ethnicities differed substantially in the mutation spectrum of the common deafness genes $[4,5]$. Nowadays, rare studies have been carried out to investigate the genetic etiology of the genes associated with hearing loss in Uyghur minority. In 2015, Chen et al. investigated the nonsyndromic sensorineural hearing loss using targeted next-generation sequencing technique in the Uyghur families, and some novel pathogenic mutations were identified in four probands including the p.L416R/p.A438T compound heterozygous mutations in TMC1, homozygous p.V1880E mutation in MYO7A, c.1238delT frame-shifting deletion in $P C D H 15$, and c.9690+1G>A splice site mutation in MYO15A. Besides, rare mutations have been identified in the hearing loss that is rarely diagnosed in the Uyghur minority [6]. Xinjiang, officially the Xinjiang Uyghur Autonomous Region, is the largest administrative division in mainland China with 


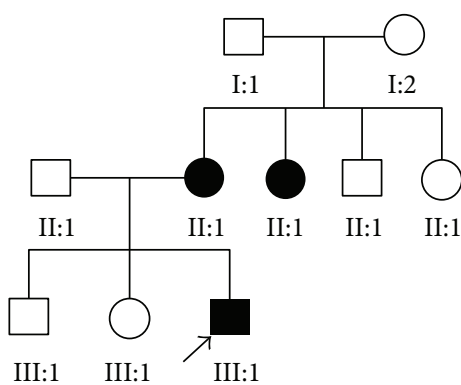

$\mathrm{JO} 2$

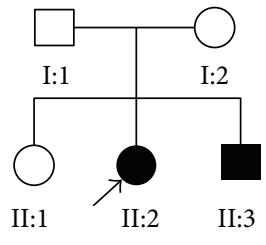

JO8

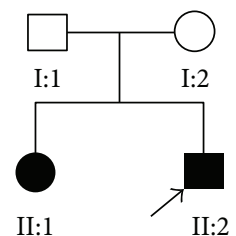

$\mathrm{J} 18$
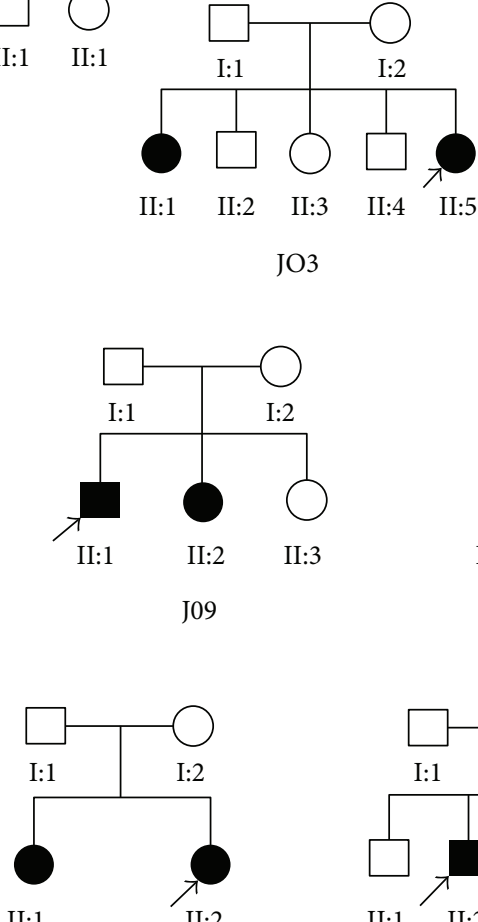

$\mathrm{J} 15$

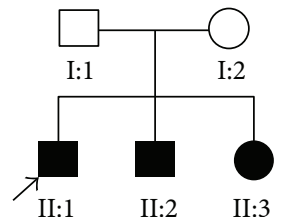

JO5
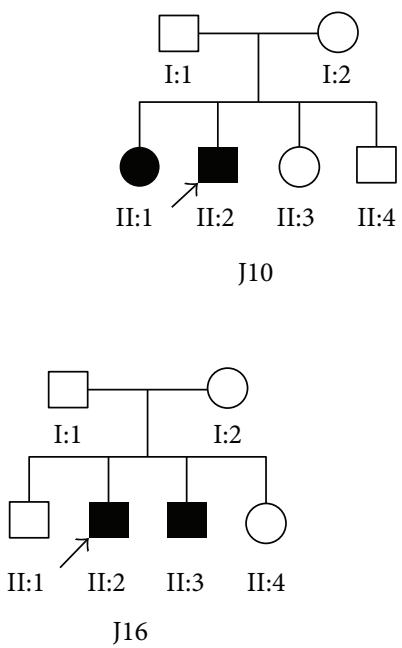
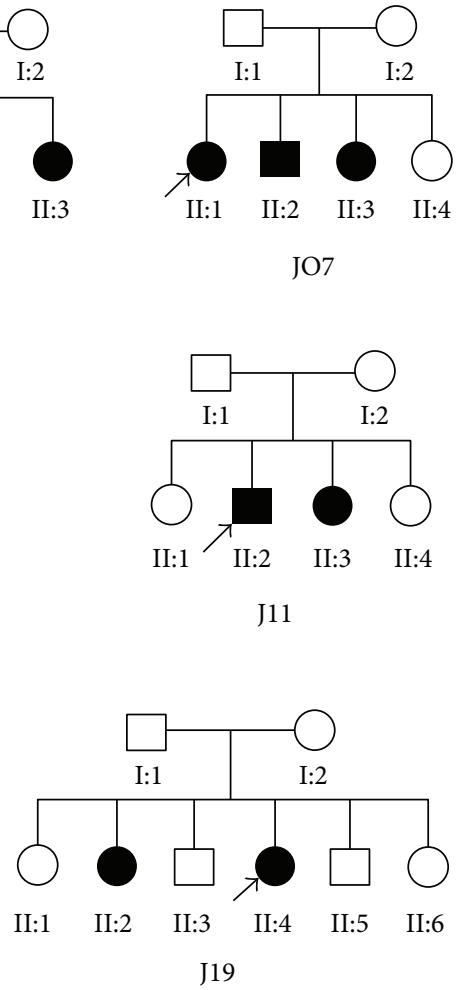

Figure 1: Pedigree of the 12 Uyghur families with hearing loss. Darkened symbols presented patients with deafness. Arrow indicated the probands.

about 10 million Uyghur habitants. Therefore, it is necessary to investigate mutations in the relatively rare deafness genes among the Uyghur families. In this study, we recruited twelve recessive Uyghur families that were excluded from mutations in common deafness genes using massively parallel DNA sequencing, to investigate the pathogenic mutations of rare deafness genes.

\section{Materials and Methods}

2.1. Subjects. Subjects from 18 Uyghur families received screening from Deaf-Mutes and Disabled Persons' Federation of Kashgar (Xinjiang, China) from March 20, 2014, to November 24, 2015, were recruited in this study. This study was approved by the Ethical Committee of the First Affiliated Hospital of Xinjiang Medical University. Written informed consent was obtained from each subject or their guardians. Eighteen unrelated Uyghur families, with two or more nonsyndromic hearing loss subjects in each family, were included in this study. Those with the possibility of environmental causes or syndromic hearing impairment were excluded from the study. All affected family members were required to receive a complete medical history inquiry and detailed physical examination (i.e., intellectual assessment, ENT routine examination, endoscopy of ear, and auditory threshold test) to exclude the possibility of environmental causes or syndromic hearing impairment. The subjects with hearing impairment were further classified based on their fourfrequency (i.e., 0.5, 1.0, 2.0, and $4.0 \mathrm{kHz}$ ) pure-tone averages (PTA) into mild (20-40 dB HL), moderate (41-55 dB HL), moderately severe (56-70 dB HL), severe (71-95 dB HL), or extremely severe $(>95 \mathrm{~dB} \mathrm{HL})$ groups, according to the WHO Hearing Classification International Standard in 2005.

2.2. Exception of Common Mutation Responsible for the Congenital Hearing Loss. Venous blood (3-5 mL) was collected from each family member, followed by DNA extraction using the commercial kit (Qiagen, Germany) according to the manufacturer's instructions. SNPscan method was used to prescreen against the 124 sites in the common deafness genes (i.e., GJB2, $12 S$ rRNA, and SLC26A4) as previously described [7]. Subsequently, subjects with SNPscan negativity were subject to massively parallel DNA sequencing for the sequencing of 97 genes known to be responsible for hearing loss. All experiments were completed in Genesky Bio-Tech Co. Ltd. (Shanghai, China) at least in triplicate.

2.3. Massively Parallel DNA Sequencing. Genome DNA was collected from two subjects with hearing loss in each family using a commercial kit (Agilent Technologies, Santa Clara, CA, USA). The genome DNA library was established according to the manufacturer's instructions, together with the 
target capture (Agilent Technologies, Santa Clara, CA, USA). All the exons, flanking introns, and splicing regions of the 97 genes were captured. Upon precise quantification, the captured DNA fragments were sequenced on Illumina HiSeq2000 analyzer. Data analysis and bioinformatics processing were performed following standard Illumina procedures.

Reads were aligned to GRCh37/hg19 assembly (https:// www.Xncbi.nlm.nih.gov/projects/genome/guide/human/ index.shtml) using the BWA software package (http://biobwa.sourceforge.net/) to identify the candidate mutations that may affect the function of the protein. Potentially pathogenic variants were defined as nonsense, missense, splice-site, and indel variants with allele frequencies of less than 0.01 in the public databases and the in-house exome database. The candidate pathogenic mutations were genotyped by Sanger sequencing in all family members. Cosegregation was performed to the mutation. The conservation of the target amino acids among the species was analyzed using the ClustalW software (http://www.genome.jp/tools-bin/clustalw).

\section{Results}

3.1. Exception of Common Mutation Responsible for the Congenital Hearing Loss. Prescreening of the 124 sites in the GJB2, $12 S$ rRNA, and SLC26A4 genes was performed in the probands of eighteen Uyghur families. Six families were excluded due to presence of common mutations. There were no common mutations among these probands of the 12 families designated as J02, J03, J05, J07, J08, J09, J10, J11, J13, J15, J16, and J19 families (Figure 1).

3.2. Patient Characteristics. Twenty-seven subjects (male: 12; female: 15) with hearing loss from 12 families were finally identified in this study, aging from 3 yrs to 30 yrs. All the subjects were confirmed with severe or extremely severe hearing loss (Table 1). All the family members showed no anomaly except one affected sibling (II:1) in the J03 family showed hearing loss combined with vision impairment and visual field defect. According to the pedigree, the features of hearing loss in all the families were in line with autosomal recessive inheritance.

3.3. Identification and Verification of Pathogenic Mutations. Eight families $(66.7 \%)$ showed biallelic mutation in the probands (Table 2), including MYO15A mutation in three families (i.e., J02, J07, and J16), MYO7A mutation in one family (J03), TMC1 mutation in two families (i.e., J09 and J11), and PCDH15 in two families (i.e., J08 and J13). Among these families, cosegregation was noticed in the mutation genes among the family members (Figures 2 and 3 ).

In the J02 family, homozygous c.6892C $>T$ (p.R2298X) in MYO15A was identified in two subjects (II:2 and III:3). Heterozygous c.6892C $>T$ in $M Y O 15 A$ was identified in I:1, I:2, and II:1 (Figure 2). In the J03 family, homozygous c.1258A >T (p.K420X) in MYO7A was identified in two siblings (II:1 and II:5). Besides, heterozygous c.1258A $>T$ in MYO7A was identified in I:1 and I:2 (Figure 2). In the J07 family, compound heterozygous c.9514C>T (p.Q3172X)/ $7894 G>T(p . V 2632 L)$ in MYO15A was identified in two
TABLE 1: General information and severity of deafness in the families.

\begin{tabular}{|c|c|c|c|c|}
\hline Family & $\begin{array}{c}\text { Patients with } \\
\text { deafness }\end{array}$ & Gender & Age (yrs) & Hearing loss \\
\hline \multirow{3}{*}{$\mathrm{J} 02$} & II:2 & Female & 30 & $\begin{array}{c}\text { Extremely } \\
\text { severe }\end{array}$ \\
\hline & II:3 & Female & 26 & Severe \\
\hline & III:3 & Male & 6 & $\begin{array}{c}\text { Extremely } \\
\text { severe }\end{array}$ \\
\hline \multirow{2}{*}{ J03 } & II: 1 & Female & 17 & $\begin{array}{c}\text { Extremely } \\
\text { severe }\end{array}$ \\
\hline & II:5 & Female & 3 & $\begin{array}{c}\text { Extremely } \\
\text { severe }\end{array}$ \\
\hline \multirow{3}{*}{ J05 } & II: 1 & Male & 18 & $\begin{array}{l}\text { Extremely } \\
\text { severe }\end{array}$ \\
\hline & II:2 & Male & 12 & $\begin{array}{l}\text { Extremely } \\
\text { severe }\end{array}$ \\
\hline & II:3 & Female & 9 & $\begin{array}{l}\text { Extremely } \\
\text { severe }\end{array}$ \\
\hline \multirow{3}{*}{ J07 } & II:1 & Female & 20 & $\begin{array}{c}\text { Extremely } \\
\text { severe }\end{array}$ \\
\hline & II:2 & Male & 19 & $\begin{array}{c}\text { Extremely } \\
\text { severe }\end{array}$ \\
\hline & II:3 & Female & 13 & Severe \\
\hline \multirow[b]{2}{*}{ J08 } & II:2 & Male & 16 & Severe \\
\hline & II:3 & Female & 12 & $\begin{array}{c}\text { Extremely } \\
\text { severe }\end{array}$ \\
\hline \multirow{2}{*}{ J09 } & II:1 & Male & 20 & Severe \\
\hline & II:2 & Female & 12 & Severe \\
\hline \multirow{2}{*}{$\mathrm{J} 10$} & II:1 & Female & 14 & Severe \\
\hline & II:2 & Male & 12 & Severe \\
\hline \multirow{2}{*}{ J11 } & II:2 & Male & 17 & $\begin{array}{c}\text { Extremely } \\
\text { severe }\end{array}$ \\
\hline & II:3 & Female & 11 & $\begin{array}{c}\text { Extremely } \\
\text { severe }\end{array}$ \\
\hline \multirow{2}{*}{$\mathrm{J} 13$} & II:1 & Female & 22 & Severe \\
\hline & II:2 & Male & 18 & Severe \\
\hline \multirow{2}{*}{ J15 } & II:1 & Female & 16 & $\begin{array}{c}\text { Extremely } \\
\text { severe }\end{array}$ \\
\hline & II:2 & Male & 13 & $\begin{array}{l}\text { Extremely } \\
\text { severe }\end{array}$ \\
\hline \multirow{2}{*}{ J16 } & II:2 & Male & 19 & Severe \\
\hline & II:3 & Male & 15 & Severe \\
\hline \multirow{2}{*}{ J19 } & II:2 & Female & 17 & Severe \\
\hline & II:4 & Female & 11 & Severe \\
\hline
\end{tabular}

siblings (II:1 and II:3). Heterozygous c.9514C $>$ T in MYO15A was identified in $\mathrm{I}: 1$, and heterozygous c.7894G $>T$ in MYO15A was identified in I:2 (Figure 2). Meanwhile, homozygous c.4658delT (p.M1553fs) in PCDH15 was identified in two siblings (II:2 and II:3) in J08 family and in two siblings (II:1 and II:2) in J13 family, respectively. In their parents (I:1 and I:2) in J08 and J13 families, heterozygous c.4658delT was identified in the PCDH15 (Figures 2 and 3). In the J09 
TABle 2: Type of mutations in the families.

\begin{tabular}{|c|c|c|c|c|c|}
\hline Type & Family & Mutation site & Amino acid changes & Type of mutation & Novel mutation \\
\hline \multirow{3}{*}{ MYO15A } & $\mathrm{J} 02$ & $6892 C>T$ & p.R2298X & Nonsense mutation & Novel \\
\hline & J07 & $9514 C>T / 7894 G>T$ & p.Q3172X/p.V2632L & Nonsense mutation/missense mutation & Novel \\
\hline & J16 & $9514 C>T$ & p.Q3172X & Nonsense mutation & Novel \\
\hline MYO7A & $\mathrm{J} 03$ & $1258 \mathrm{~A}>\mathrm{T}$ & p.K420X & Nonsense mutation & Novel \\
\hline \multirow{2}{*}{ TMC1 } & J09 & $773 G>A$ & p.G258D & Missense mutation & Novel \\
\hline & J11 & $1247 T>G / 1312 G>A$ & p.L416R/p.A438T & Missense mutation & Reported previously \\
\hline \multirow{2}{*}{ PCDH15 } & J08 & 4658delT & p.M1553fs & Frameshift mutation & Novel \\
\hline & J13 & 4658delT & p.M1553fs & Frameshift mutation & Novel \\
\hline
\end{tabular}

family, the homozygous $c .773 G>A(p . G 258 D)$ was noticed in the TMC1 gene in two siblings (II:1 and II:2). Heterozygous c.773G $>A$ in TMC1 was identified in I:1 and I:2 (Figure 3). In the J11 family, the compound heterozygous $c .1247 T>G$ / $1312 G>A$ was identified in the TMC1 gene in two siblings (II:2 and II:3). Sanger sequencing was performed to their parents (I:1 and I:2), which revealed c.1247T>G in I:1 and c. $1312 G>A$ in I:2 responsible for the p.L416R and p.A438T mutations, respectively (Figure 3). In J16 family, the homozygous c.9514C>T (p.Q3172X) was identified in the MYO15A in two siblings (II:2 and II:3). Heterozygous $c .9514 C>T$ in MYO15A was identified in I:1 and I:2 (Figure 3). The missense mutations of p.V2632L in MYO15A and p.G258D, p.L416R, and p.A438T in TMC1 were highly conserved among various species (Figure 4). No pathogenic mutations were noticed in the other families including J05, J10, J15, and $\mathrm{J} 19$, respectively.

\section{Discussion}

Rare studies have been carried out to investigate the molecular etiology of hearing loss in the Uyghur minority as it shows lower incidence compared with that of the Han Chinese [8]. In this study, massively parallel DNA sequencing was used to screen rare deafness genes in the Uyghur families, and several new mutations that were suggested to be related to the pathogenesis of the disease were identified. This study is helpful to increase our understanding on the molecular etiology of hearing loss in Uyghur minority.

Mutations of MYO15A at the DFNB3 locus appear to be the third or fourth most common cause of autosomal recessive nonsyndromic deafness [9]. Myosin 15A is mainly expressed in the cochlea and plays important roles in the differentiation and extension of the stereocilium in the hair cells [10]. Up to now, 48 types of mutation have been reported particularly in the subjects in Pakistan, Turkey, and Iran $[8,11,12]$. In this study, homozygous and heterozygous MYO15A mutations were identified in the J02, J07, and J16 families, and their parents were carriers of mutation. In the J02 and J16 families, nonsense mutation was identified in each family, which finally resulted in impairment of protein function and the consequent phenotypes of hearing loss. Nonsense mutation was responsible for extremely severe hearing loss. Our results indicated that subjects with newly identified nonsense mutations (p.R2298X/p.Q3172) of
MYO15A in the J02 and J16 families showed severe or extremely severe hearing loss according to the threefrequency PTA. Additionally, two newly identified mutations (e.g., p.Q3172X/p.V2632L) of MYO15A were noticed in the J07 family, and the subjects presented severe hearing loss. These results were in line with the fact that MYO15A mutation may be associated with severe or extremely severe hearing loss among subjects $[13,14]$.

TMC1 encodes a transmembrane protein (TMC1 protein). TMC1 mutation was reported to induce both autosomal dominant and recessive hearing loss (DFNA36 and DFNB7/B11) in a large number of populations. To date, a total of 52 mutations have been reported in TMC1 gene [15]. In this study, homozygous mutation and compound heterozygous mutation were identified in the TMC1 gene in the J09 and J11 families, with the parents as the mutation carriers. To be exact, missense mutations (p.G258D/p.G258D and p.L416R/p.A438T) were identified in the J09 and J11 families. These sequence mutations in amino acid were highly conserved among the species. On this basis, we speculated that the mutations may induce function loss of the encoded proteins, which affects the ion channel formation on the surface of the hair cells in the internal ear. The transmission of the potassium ion was hampered, which consequently led to dysfunction of cochlear hair cells [16]. Among these mutations, p.L416R/p.A438T compound heterozygous mutations in TMC1 were considered as pathogenic mutations in Uyghur families [6]. A novel p.G258D was identified in one family in this study. Autosomal recessive hereditary deafness caused by TMC1 gene was mainly featured by severe or extremely severe congenital sensorineural deafness. In line with the previous study, the patient showed clinical manifestations of severe congenital deafness in the J09 family, while the patient showed manifestations of extremely severe congenital deafness in the J11 family. Whereas, some patients may present phenotype of severe sensorineural hearing loss $[17,18]$.

Mutations of $P C D H 15$, encoding protocadherin 15, are responsible for inducing combined hearing and vision impairment (type 1 Usher syndrome; USH1F) or nonsyndromic deafness (DFNB23) [19]. Human PCDH15 is expressed in the cochlea, the external synapse of the optic nerves, and the retinal cells. To the best of our knowledge, PCDH15 mutation could induce nonsyndromic hearing loss and Usher syndrome type 1F. The major difference between 


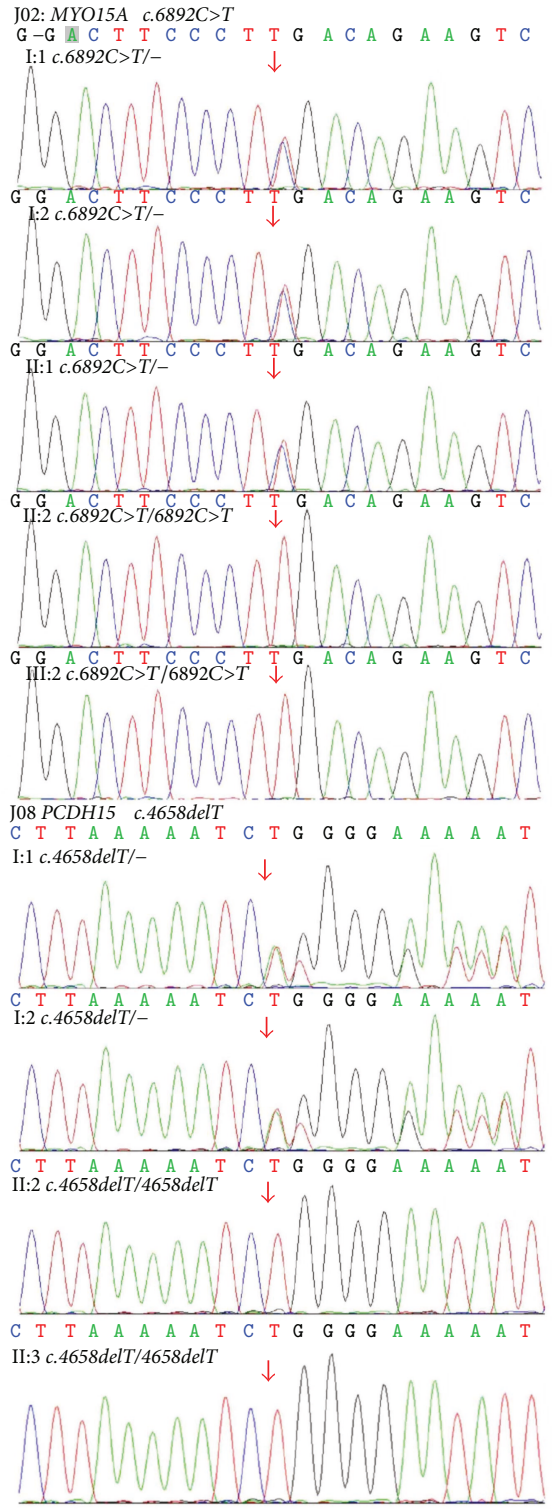

J03 MYOTA $c .1258 A>T$
A G C A A T T T T A C A A A G C C C C T C C C C
1. T C C

$\begin{array}{lllllll}\text { I: } c .1258 A>T /- & & & \downarrow\end{array}$
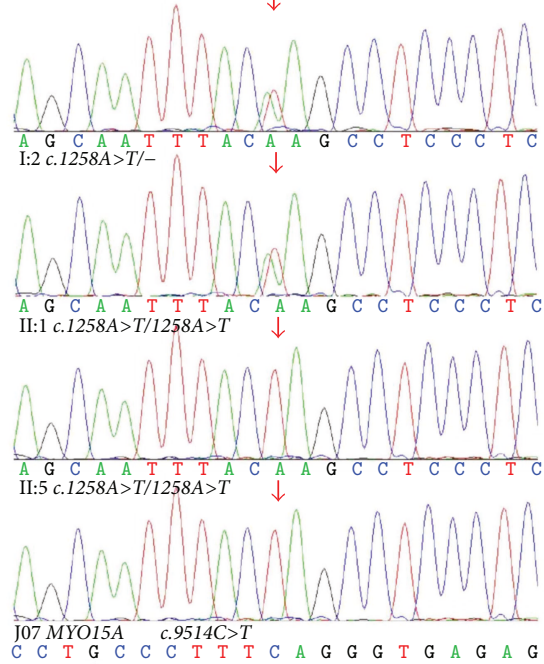
J07 MYO15A c.9514C>

$C$ C T G C C C I I I C A G G G I G A G A G $\mathrm{I}: 1$ c. $9514 C>T /-$

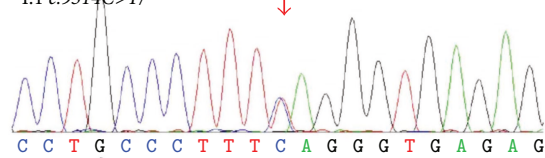
I: 2 WT

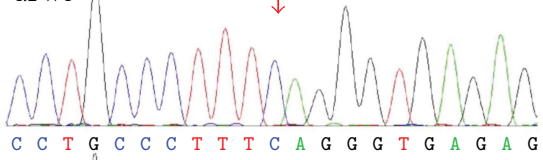

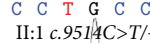

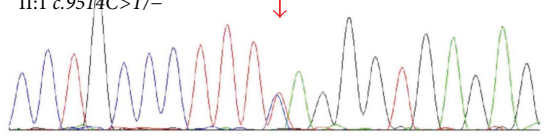

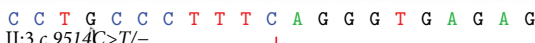
II:3 c.9514C $>$ T/ $-\quad \downarrow$

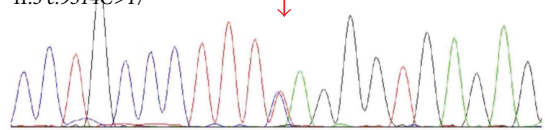

J07 MYO15A c.7894G>T

G G T G G G A C A G G G T G I:1 $W T$

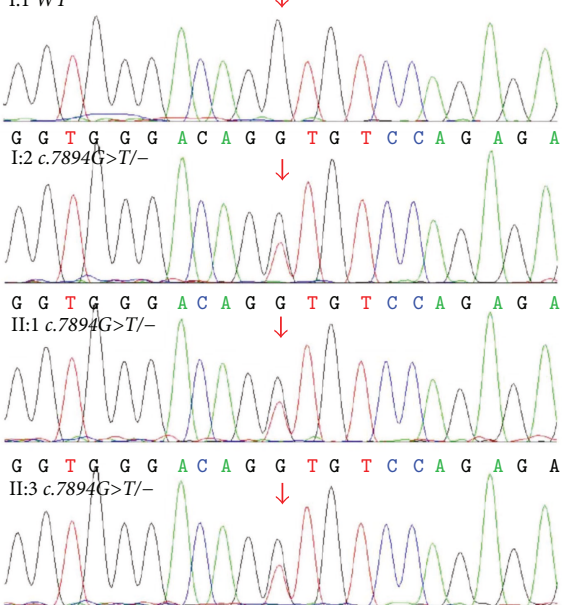

FigURE 2: Sequencing results of the J02, J03, J07, and J08 families.

these conditions is the presence of vision loss [20]. The relationship between the genotype and phenotype of PCHD15 was closely related to the mutation type [21]. For example, frameshift mutation or nonsense mutation may induce syndromic hearing loss. For the missense mutation, the hearing rather than visual acuity may be hampered due to mutation, 

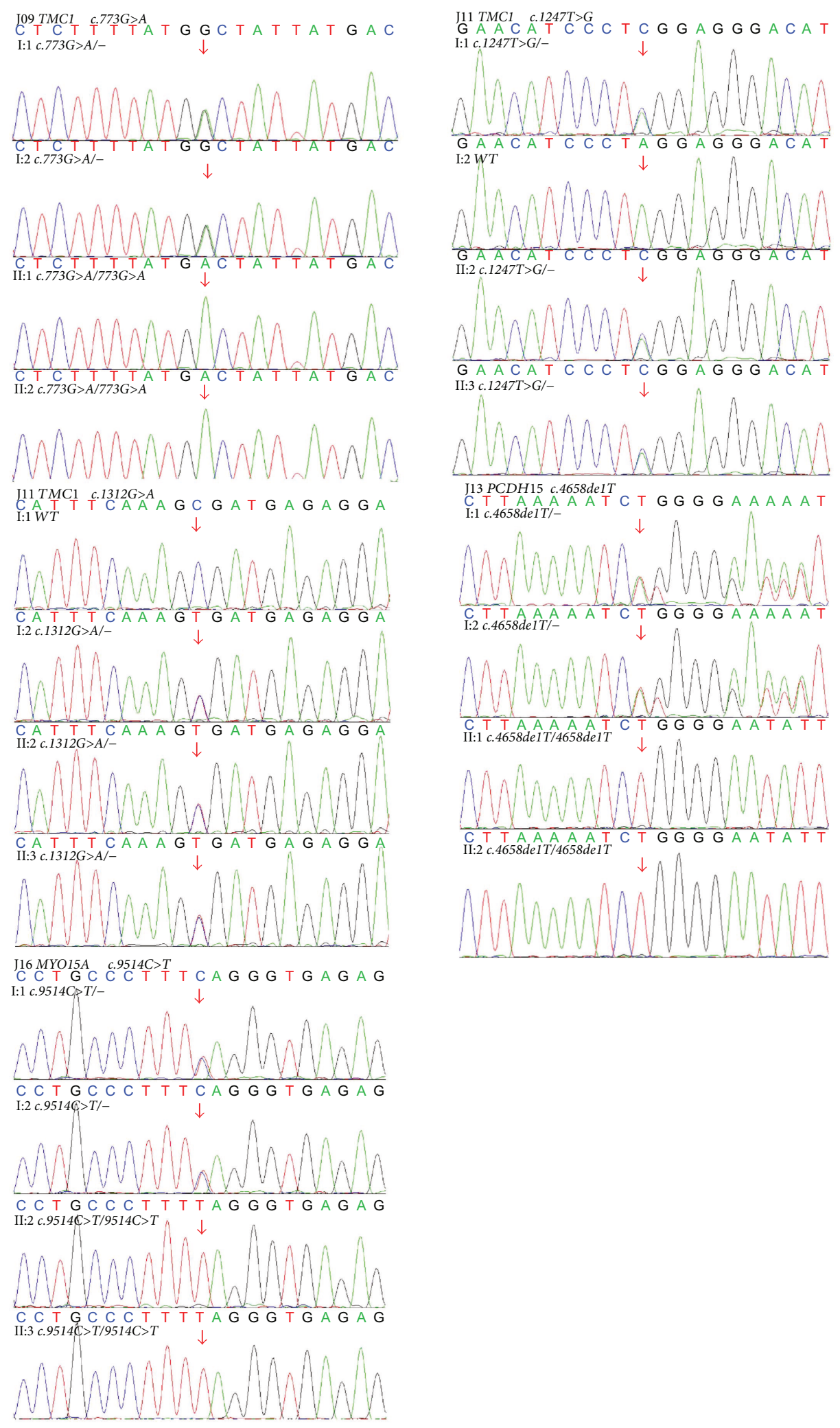

FigURE 3: Sequencing results of the J09, J11, J13, and J16 families.

which finally lead to syndromic hearing loss $[22,23]$. In this study, a newly identified frameshift mutation (p.M1553fs) was noticed in the J08 and J13 families. We speculated that it might induce structural changes of encoding protein together with tip link of the hair cells in the inner ear and finally lead to hearing loss. The patients (aged 12-22 yrs) showed severe or extremely severe hearing loss. Despite frameshift mutations were identified in these patients, no 


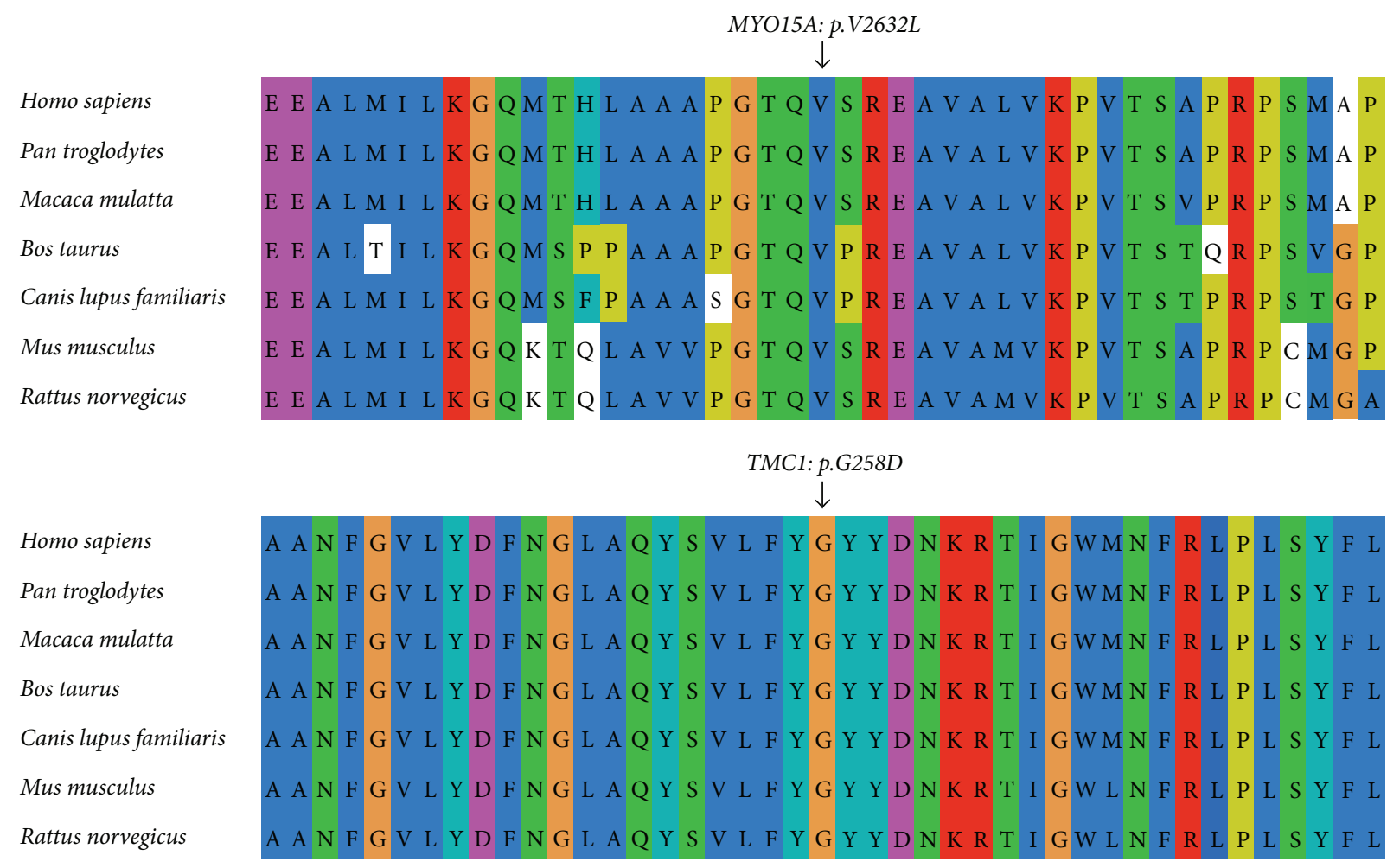

TMC1: p.A438T

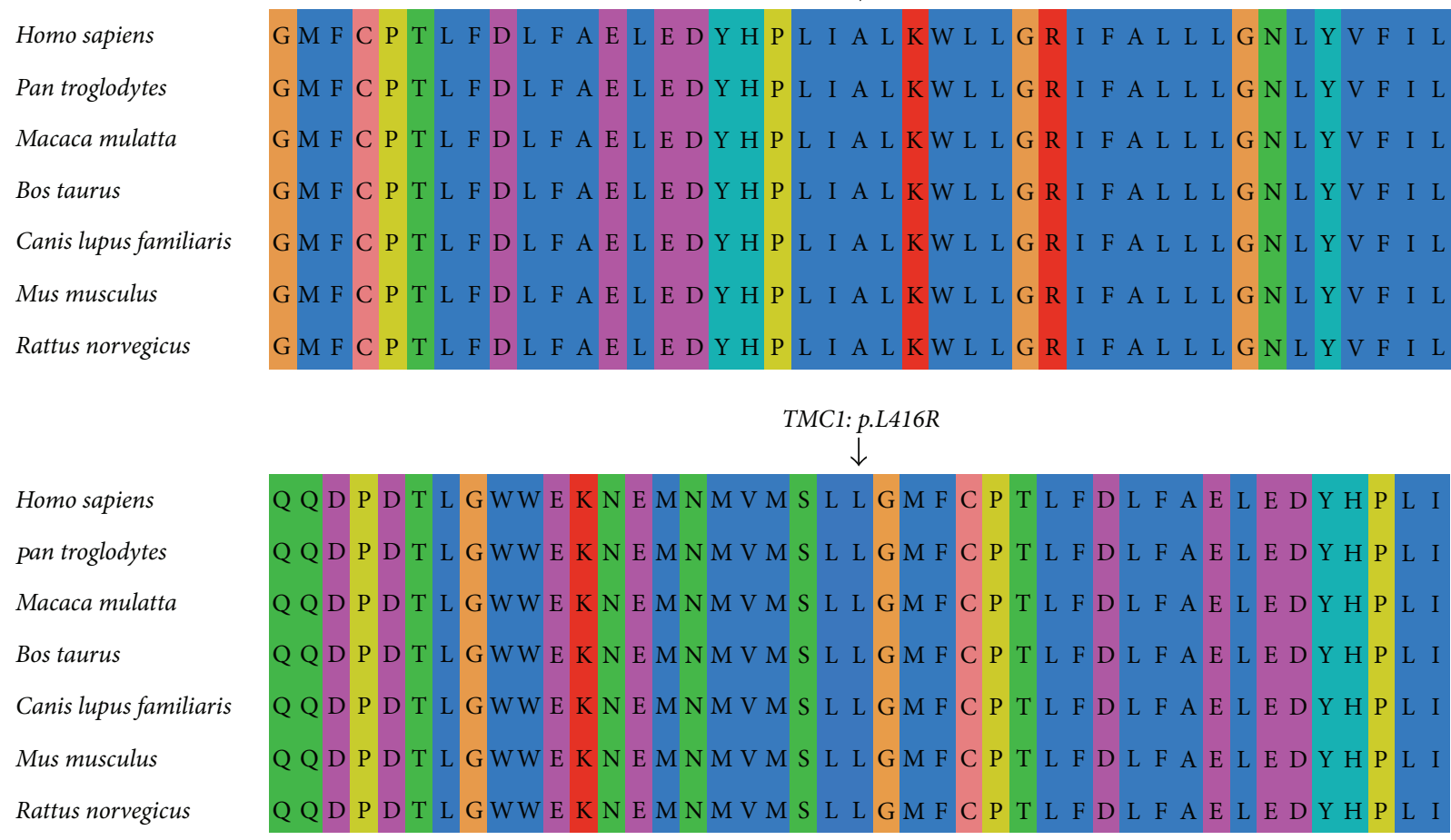

FIGURE 4: The missense mutations of p.V2632L in MYO15A and p.G258D, p.L416R, and p.A438T in TMC1 in multiple species after sequencing.

visual disorder featured by night blindness was observed with the aging of the patients. Besides, in a consanguineous Pakistani family, missense mutation of $\mathrm{PCDH} 15$ was reported to be responsible for inducing Usher syndrome IF type. On this basis, the relationship between phenotype and genotype of $P C D H 15$ is still not well defined. Further observations should be paid to the occurrence of delayed visual disorder in the patients of J08 and J13 families. 
The MYO7A gene encodes the actin-binding motor protein myosin VIIa. The myosin VIIa protein is expressed in the cochlea, cytoplasm of outer hair cells, stereocilium, retinal pigment epithelial cells, photoreceptor cells, and epithelial cells on the vestibular nerves [24]. To date, more than 160 different mutations of $M Y O 7 A$ gene have been identified, most of which have been reported to induce Usher syndrome type IB and nonsyndromic Usher syndrome $[25,26]$. Part of the mutations has been reported to be associated with nonsyndromic hearing loss (DFNAII and DFNB2) [25]. On this basis, it is reasonable to speculate that there might be sharp differences in the variance of phenotype after mutation. Similar with $P C D H 15$, the difference of syndromic and nonsyndromic hearing loss induced by mutation of $M Y O 7 A$ was visual disorder. In cases of MYO7A mutation, the function of MYO7A protein in the retina may be compensated by the protein with similar function or by the residue wild-type dimer. Otherwise, vision loss may be induced as no adequate compensation of the protein function in the retina by the wild-type dimer. Nevertheless, the function of MYO7A protein could not be compensated in the internal ear, which led to functional loss [26].

USH1B and USH1F were featured by constriction of visual field, visual disturbance with or without vestibular dysfunction caused by progressive retinitis pigmentosa $[27,28]$. In this study, a novel nonsense mutation $(p . K 420 X)$ was noticed in MYO7A in the J03 family, which may induce hearing loss by changing the protein structures. Two siblings (II:5, 6 yrs, with no visual disorder; II:1, 17 yrs) in the family showed severe congenital hearing loss. For II:1, the patient presented visual disorder at the age of 7, which showed gradual deterioration together with obvious light blindness and spot-like defect of visual field. No vestibular disorder was noticed. For the II:5, no visual disorders were noticed as the patient was at the young age. The phenotypes of the II:1 and II:5 were different despite the same mutation in MYO7A, which were manifested as DFNB2 and USH1B, respectively. In the future, close observations should be paid to the visual disorder of the II:5 to confirm whether she suffers from USH1B or not.

Indeed, there are some limitations in this study. Actually, the sample size is not large enough. In addition, we are not sure whether the identified mutations can represent the whole Uyghur population with hearing loss in mainland China. In the future, we will focus on large sample studies to identify the roles of these mutations in the pathogenesis of hearing loss.

In conclusion, mutations in rare hearing loss genes were noticed in 8 Uyghur families using massively parallel DNA sequencing. In total, 6 novel types of mutation were identified including $6892 C>T, 9514 C>T / 7894 G>T$, and $9514 C>T$ in MYO15A gene (J02, J07, and J16 families), $1258 A>T$ in MYO7A (J03 family), 773G $>A$ in TMC1 (J09 family), and 4658delT in PCDH15 (J08 and J13 families). Besides, the ratio of nonsense mutation and frameshift mutation was comparatively high, which indicated that the mutation types were rare. The homozygous mutations in the Uyghur families were higher in incidence. Our study contributed to the investigation of molecular etiology of hearing loss in Uyghur minority.

\section{Abbreviations}

ARNSHL: Autosomal recessive nonsyndromic hearing loss PTA: $\quad$ Pure-tone averages.

\section{Conflicts of Interest}

The authors declare that they have no conflicts of interest.

\section{Acknowledgments}

This study was supported by the National Natural Science Foundation of China (no. 81360158).

\section{References}

[1] S. Shafique, S. Siddiqi, M. Schraders et al., "Genetic spectrum of autosomal recessive non-syndromic hearing loss in Pakistani families," PLoS One, vol. 9, no. 6, article e100146, 2014.

[2] P. Dai, F. Yu, B. Han et al., "GJB2 mutation spectrum in 2063 Chinese patients with nonsyndromic hearing impairment," Journal of Translational Medicine, vol. 7, no. 1, p. 26, 2009.

[3] S. Reyes, G. Wang, X. Ouyang et al., "Mutation analysis of SLC26A4 in mainland Chinese patients with enlarged vestibular aqueduct," Otolaryngology and Head and Neck Surgery, vol. 141, no. 4, pp. 502-508, 2009.

[4] Y. Chen, M. Tudi, J. Sun et al., "Genetic mutations in nonsyndromic deafness patients of Uyghur and Han Chinese ethnicities in Xinjiang, China: a comparative study," Journal of Translational Medicine, vol. 9, no. 1, p. 154, 2011.

[5] W. Du, Q. Wang, Y. Zhu, Y. Wang, and Y. Guo, “Associations between GJB2, mitochondrial 12S rRNA, SLC26A4 mutations, and hearing loss among three ethnicities," BioMed Research International, vol. 2014, Article ID 746838, 6 pages, 2014.

[6] Y. Chen, Z. Wang, Z. Wang et al., "Targeted next-generation sequencing in Uyghur families with non-syndromic sensorineural hearing loss," PLoS One, vol. 10, no. 5, article e0127879, 2015.

[7] H. Li, B. Wang, D. Liu et al., "SNPscan as a high-performance screening tool for mutation hotspots of hearing loss-associated genes," Genomics, vol. 106, no. 2, pp. 83-87, 2015.

[8] X. Gao, Q. Y. Zhu, Y. S. Song et al., "Novel compound heterozygous mutations in the MYO15A gene in autosomal recessive hearing loss identified by whole-exome sequencing," Journal of Translational Medicine, vol. 11, no. 1, p. 284, 2013.

[9] M. B. Petersen and P. J. Willems, "Non-syndromic, autosomal-recessive deafness," Clinical Genetics, vol. 69, no. 5, pp. 371-392, 2006.

[10] A. Wang, Y. Liang, R. A. Fridell et al., "Association of unconventional myosin MYO15 mutations with human nonsyndromic deafness DFNB3," Science, vol. 280, no. 5368, pp. 1447-1451, 1998.

[11] D. Duman, A. Sirmaci, F. B. Cengiz, H. Ozdag, and M. Tekin, "Screening of 38 genes identifies mutations in $62 \%$ of families with nonsyndromic deafness in Turkey," Genetic Testing and Molecular Biomarkers, vol. 15, no. 1-2, pp. 29-33, 2011.

[12] Z. Fattahi, A. E. Shearer, M. Babanejad et al., "Screening for MYO15A gene mutations in autosomal recessive nonsyndromic, GJB2 negative Iranian deaf population," American Journal of Medical Genetics A, vol. 158A, no. 8, pp. 1857$1864,2012$. 
[13] W. Li, L. Guo, Y. Li et al., "A novel recessive truncating mutation in MYO15A causing prelingual sensorineural hearing loss," International Journal of Pediatric Otorhinolaryngology, vol. 81, pp. 92-95, 2016.

[14] H. Xia, X. Huang, Y. Guo et al., "Identification of a novel MYO15A mutation in a Chinese family with autosomal recessive nonsyndromic hearing loss," PLoS One, vol. 10, no. 8, article e0136306, 2015.

[15] A. Bakhchane, H. Charoute, H. Nahili et al., "A novel mutation in the TMC1 gene causes non-syndromic hearing loss in a Moroccan family," Gene, vol. 574, no. 1, pp. 28-33, 2015.

[16] X. Gao, Y. Su, L. P. Guan et al., "Novel compound heterozygous TMC1 mutations associated with autosomal recessive hearing loss in a Chinese family," PLoS One, vol. 8, no. 5, article e63026, 2013.

[17] A. Imtiaz, A. Maqsood, A. U. Rehman et al., "Recessive mutations of TMC1 associated with moderate to severe hearing loss," Neurogenetics, vol. 17, no. 2, pp. 115-123, 2016.

[18] J. Hu, F. Liu, W. Xia et al., "Exome sequencing identifies a mutation in TMC1 as a novel cause of autosomal recessive nonsyndromic hearing loss," Journal of Translational Medicine, vol. 14, no. 1, p. 29, 2016.

[19] Z. M. Ahmed, S. Riazuddin, S. L. Bernstein et al., "Mutations of the protocadherin gene $\mathrm{PCDH} 15$ cause Usher syndrome type 1F," American Journal of Human Genetics, vol. 69, no. 1, pp. 25-34, 2001.

[20] Z. M. Ahmed, S. Riazuddin, S. Aye et al., "Gene structure and mutant alleles of PCDH15: nonsyndromic deafness DFNB23 and type 1 Usher syndrome," Human Genetics, vol. 124, no. 3, pp. 215-223, 2008.

[21] Z. M. Ahmed, S. Riazuddin, J. Ahmad et al., " $P C D H 15$ is expressed in the neurosensory epithelium of the eye and ear and mutant alleles are responsible for both USH1F and DFNB23," Human Molecular Genetics, vol. 12, no. 24, pp. 3215-3223, 2003.

[22] T. Jaijo, A. Oshima, E. Aller et al., "Mutation screening of the PCDH15 gene in Spanish patients with Usher syndrome type I," Molecular Vision, vol. 18, pp. 1719-1726, 2012.

[23] H. Yoshimura, M. Miyagawa, K. Kumakawa, S. Y. Nishio, and S. Usami, "Frequency of Usher syndrome type 1 in deaf children by massively parallel DNA sequencing," Journal of Human Genetics, vol. 61, no. 5, pp. 419-422, 2016.

[24] L. M. Astuto, M. D. Weston, C. A. Carney et al., "Genetic heterogeneity of Usher syndrome: analysis of 151 families with Usher type I," American Journal of Human Genetics, vol. 67, no. 6, pp. 1569-1574, 2000.

[25] Y. Ma, Y. Xiao, F. Zhang et al., "Novel compound heterozygous mutations in MYO7A gene associated with autosomal recessive sensorineural hearing loss in a Chinese family," International Journal of Pediatric Otorhinolaryngology, vol. 83, pp. 179-185, 2016.

[26] M. W. Luijendijk, E. Van Wijk, A. M. Bischoff et al., "Identification and molecular modelling of a mutation in the motor head domain of myosin VIIA in a family with autosomal dominant hearing impairment (DFNA11)," Human Genetics, vol. 115, no. 2, pp. 149-156, 2004.
[27] X. Gao, G. J. Wang, Y. Y. Yuan et al., "Novel compound heterozygous mutations in MYO7A Associated with Usher syndrome 1 in a Chinese family," PLoS One, vol. 9, no. 7, article e103415, 2014.

[28] H. Yoshimura, S. Iwasaki, S. Y. Nishio et al., "Massively parallel DNA sequencing facilitates diagnosis of patients with Usher syndrome type 1," PLoS One, vol. 9, no. 3, article e90688, 2014. 


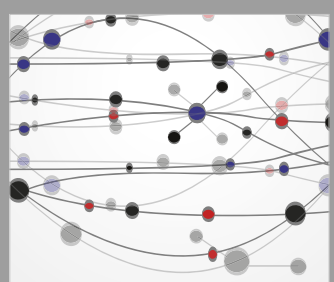

The Scientific World Journal
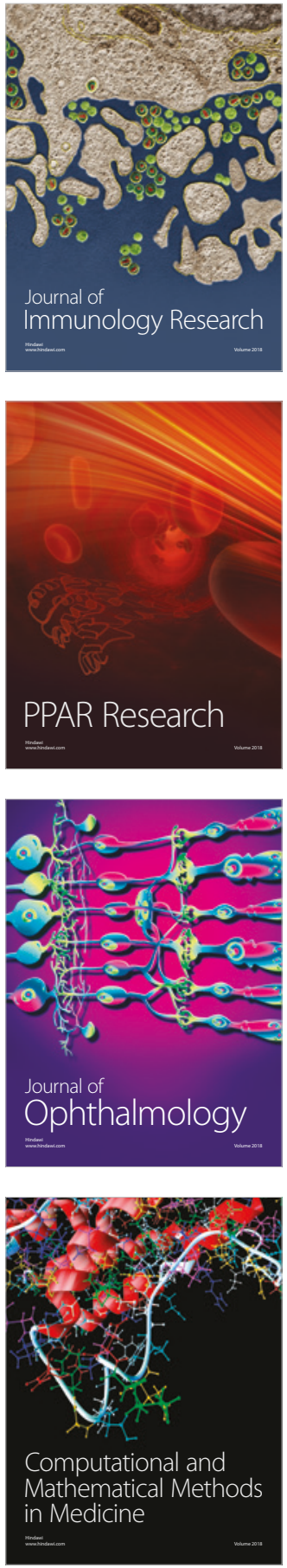

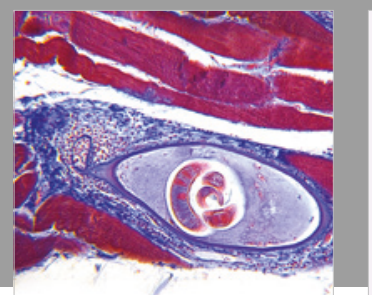

Gastroenterology Research and Practice

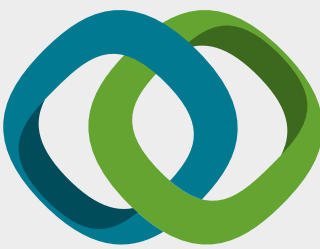

\section{Hindawi}

Submit your manuscripts at

www.hindawi.com
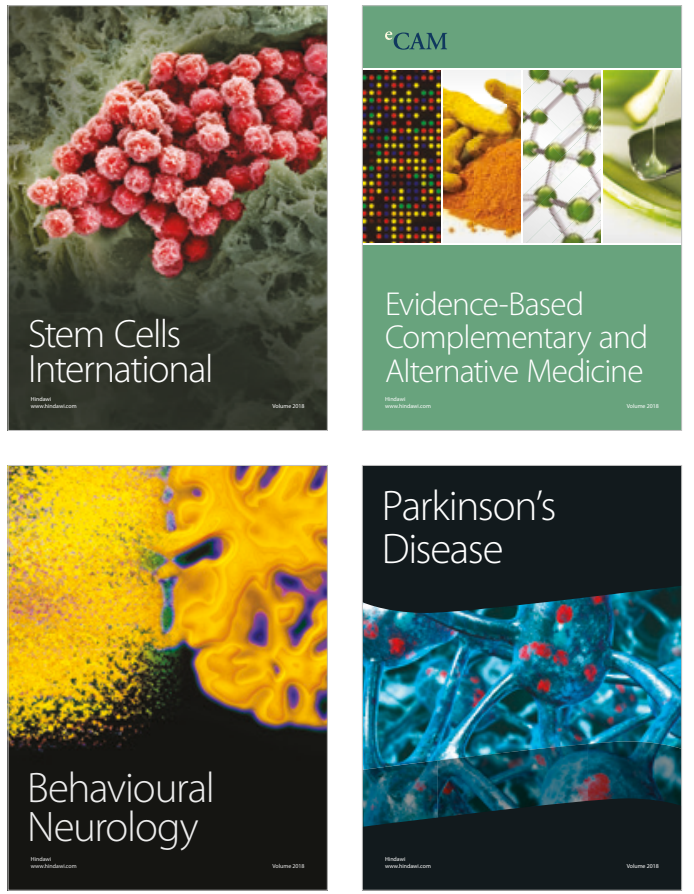

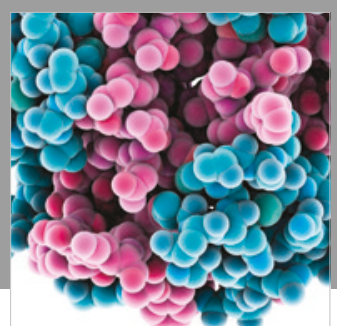

ournal of

Diabetes Research

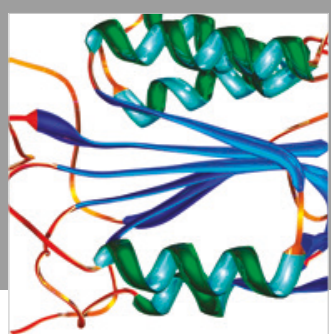

Disease Markers
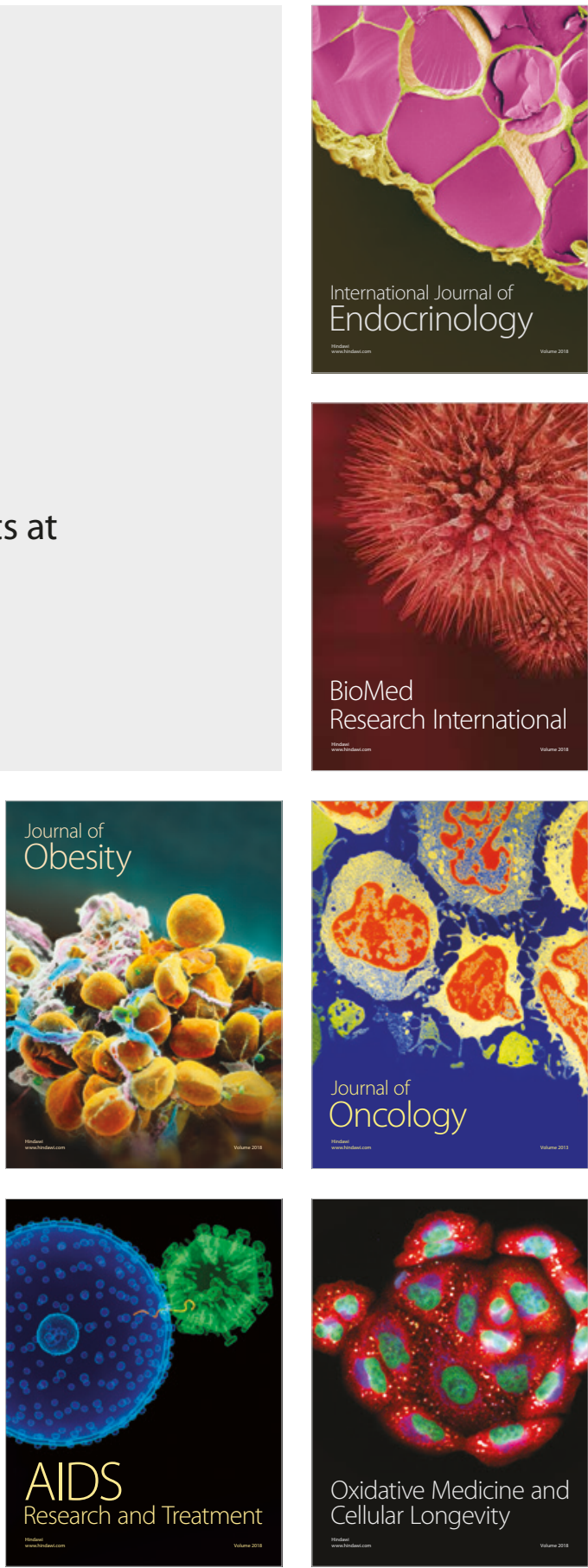